BULLETIN OF THE

AMERICAN MATHEMATICAL SOCIETY

Volume 79, Number 3, May 1973

\title{
NONLOCALLY FLAT EMBEDDINGS, SMOOTHINGS, AND GROUP ACTIONS
}

\author{
BY SYLVAIN E. CAPPELL ${ }^{1}$ AND JULIUS L. SHANESON ${ }^{1}$ \\ Communicated by William Browder, October 12, 1972
}

Introduction. This note announces some methods and results concerned with piecewise linear (PL) embeddings of manifolds, in codimension two, that are not necessarily locally flat, i.e., not locally smoothable. Theorems 4 and 1 are PL embedding theorems in codimension two. They are analogous to $\mathrm{PL}$ embedding theorems of $[\mathbf{H}]$ (see also [W], especially for nonsimply connected cases) for higher codimensions; recall that for codimension greater than two, a PL embedding of a manifold is always locally flat. The present results have a simpler form in odd dimensions.

Our methods can also be applied to the study of equivariant submanifolds, as in Theorems 2 and 3 below. In the papers [CS3], [CS4], it was determined which free actions of a cyclic group $Z_{p}$ on a sphere $S^{2 n-1}$ are the restrictions of a given free $Z_{p}$ action $T$ on $S^{2 n+1}$ to an invariant sphere for which the action carries the fibers of a closed tubular neighborhood linearly to fibers. Using a new embedding theorem, it is determined here which free cyclic actions on $S^{2 n-1}, n \geqq 2$, are restrictions of $T$ to an invariant sphere, without any condition on the behavior of the action in the neighborhood of the invariant sphere.

The proof of Theorem 3 leads to examples of the following type: Let $n \geqq 4$ be an integer not of the form $2^{r}-1$ or $2^{r}-3$, and let $P^{n}$ denote real projective $n$-space. Then there is a PL manifold $Q^{n+2}$, homotopy equivalent to $P^{n+2}$, with the following properties:

(i) There are PL embeddings of $P^{n}$ in $Q^{n+2}$.

(ii) If $f: P^{n} \rightarrow Q^{n+2}$ is a PL embedding, let $X_{f} \subset P^{n}$ be the set of points at which $f$ is not locally flat. Then the natural map $H_{i}\left(X_{f} ; Z_{2}\right) \rightarrow H_{i}\left(P^{n} ; Z_{2}\right)$ is onto for $0 \leqq i \leqq n-2$.

Singularities of PL embeddings will be studied further in [CS5].

We also compute the PL, but not necessarily locally flat, $s$-cobordism classes of PL embeddings, homotopic to the zero-section, of a manifold in a disk bundle. For example, for the $n$-torus $T^{n}=S^{1} \times \cdots \times S^{1}$, $n \geqq 3$, the set of such classes is in one-to-one correspondence with a finite nontrivial direct sum of subgroups of knot cobordism groups consisting of knots with vanishing index or Arf invariant. In contrast, it was

AMS (MOS) subject classifications (1970). Primary 57A40, 57A45, 57C35, 57C40, 57C45, 57E10; Secondary 55A25, 57C25, 57D65.

${ }^{1}$ Both authors are A. P. Sloan fellows and are partially supported by NSF grants. 
shown in [CS3], [CS4] that for $n$ odd, for example, the group $C_{\mathrm{PL}}\left(T^{n}\right)$ of PL locally flat cobordism classes of embeddings of $T^{n}$ in $T^{n} \times D^{2}$, homotopic to the zero-section, is a finite sum of copies of the quotients of knot cobordism groups by the subgroups of knots of vanishing index or Arf invariant. Thus the group $C_{\mathrm{PL}}\left(T^{n}\right)$ is a finite sum of copies of $Z$ and $Z_{2}$.

For a PL manifold $W^{n+2}$ of the homotopy type of a PL $n$-manifold, $n \geqq 3$, we show that if $n$ is odd, or if $\pi_{1} W$ vanishes or is infinite cyclic ${ }^{2}$ and $W$ is orientable, then every closed manifold $M$ (assumed orientable if $\pi_{1} W^{2 k}=Z$ ) homotopy equivalent to $W$ occurs as a spine of $W$; i.e., $M$ can be PL embedded in $W$ by a simple homotopy equivalence, so that $W$ will be a regular neighborhood of the image. One consequence is the result of Kato-Matsumoto [KM] on the existence, in the simply-connected case, of a spine that is locally flat, for $n$ odd, and locally flat except at one point, for $n$ even. (This result also follows easily from the codimension two splitting theorems of [CS4, §8].) On the other hand, given $W$ as above one can find a homotopy equivalence $h: M \rightarrow W$ with the following properties: (i) $h$ is homotopic to a PL embedding, and (ii) for every embedding $f: W \rightarrow M$ homotopic to $h$, the set $X_{f}$ of nonlocally flat points is large in the sense that $X_{f} \cap F\left(V_{\alpha}\right)$ is nonempty for every component $V_{\alpha}$ of a characteristic variety of Sullivan [S2], $F: V \rightarrow M$ of $M$.

Statement of some results. Below all manifolds are PL, connected, and compact, and all embeddings are PL, i.e., simplicial after suitable subdivision, but not necessarily locally flat. Related topological results will be discussed elsewhere. More results and proofs will appear in [CS5].

THEOREM 1. Let $f: N^{n} \rightarrow W^{n+2}$ be a PL embedding and let $g: M \rightarrow N$ be a homotopy equivalence of closed PL manifolds. Assume that $n$ is odd, or that $\pi_{1} N$ vanishes, or that $\pi_{1} N$ is infinite cyclic and $N$ and $W$ are orientable., Then $\mathrm{fg}$ is homotopic to a PL embedding.

(It suffices for Theorem 1 that $N$ be a PL embedded Poincaré complex, $n \neq 2$.)

THEOREM 2. Let $\left(\Sigma^{2 n-1}, T_{1}\right),\left(\Sigma^{2 n+1}, T_{2}\right), n \neq 2$, be spheres, equipped with free $P L$ actions of $S^{1}, T_{1}$, and $T_{2}$. Then there is an equivariant $P L$ embedding of $\left(\Sigma^{2 n-1}, T_{1}\right)$ in $\left(\Sigma^{2 n+1}, T_{2}\right)$.

However, an embedding that induces a linear action of $S^{1}$ on the normal bundle exists, for $n \geqq 3$, if and only if $\left(\Sigma^{2 n+1}, T_{2}\right)$ is a suspension of $\left(\Sigma^{2 n-1}, T_{1}\right)$, which will not in general be the case [S2].

\footnotetext{
${ }^{2}$ This uses the homology splitting principle of $[$ CS4, V] for $\Gamma$-groups.

${ }^{3}$ The result remains valid if $\pi_{1}(R-N)$ is abelian, $R$ a regular neighborhood of $N$ in $W$.

${ }^{4}$ That is, the quotient space is a PL manifold.
} 
THEOREM 3. Let $\left(\Sigma_{1}^{2 n-1}, T_{1}\right)$ and $\left(\Sigma_{2}^{2 n+1}, T_{2}\right)$ be spheres equipped with free $P L$ actions of $Z_{p}, T_{1}$ and $T_{2}$. Then there is an equivariant embedding of $\left(\Sigma_{1}, T_{1}\right)$ in $\left(\Sigma_{2}, T_{2}\right)$ if and only if $\exists$ a suspension $\left(\Sigma_{0}, T_{0}\right)$ of $T_{1}$ (see [W]) with $\Sigma_{0} / T_{0}$ homotopy equivalent to $\Sigma^{2 n+1} / T_{2}$. However, for $p$ odd, this embedding could be constructed so that $T_{2}$ restricts to a linear $Z_{p}$ action on the normal bundle of $\Sigma_{1}^{2 n-1}$ in $\Sigma_{2}^{2 n+1}$ if and only if $\Sigma_{1} / T_{1}$ is normally cobordant to a desuspension of $\Sigma_{2} / T_{2}, n \geqq 3$ [CS3], [CS4].

The more complicated situation for $p$ even is studied in [CS3], [CS4]. (See also [CS6].)

To illustrate the main ideas involved in proving theorems such as the above, we first introduce the notion of a (homology) Poincaré embedding in codimension two. By a Poincare embedding of the closed manifold $M^{n}$ in the closed manifold $W^{n+2}$ we mean a (simple) homotopy equivalence $f: W \rightarrow(E(\xi) \cup \dot{E}(\xi) Y)$, where $E(\xi)$ is the total space of the associated disk fibration of a spherical fiber space $\xi$ over $M$, and where $(Y, E(\xi))$ is a Poincare pair (even only with coefficients in $Z \pi_{1} W$ ). We say $f$ is realized by a PL embedding if there are PL manifolds $(V, \partial V),(X, \partial X), \partial X=\partial V$, equipped with a PL embedding $M \rightarrow V$; a homology equivalence, inducing an isomorphism on $\pi_{1}$, with coefficients in the local system of $\pi_{1} M,(V, \partial V) \rightarrow(E(\xi), \dot{E}(\xi))$; and a homology equivalence with coefficients in the local system of $\pi_{1}(W),(X, \partial X) \rightarrow(Y, \dot{E}(\xi))$; so that the diagrams
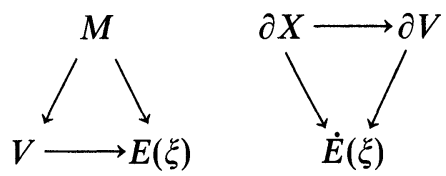

are homotopy commutative and so that the induced map $\left(V \cup_{\partial V} X\right) \rightarrow W$ is homotopic to a PL homeomorphism. These notions can be relativized if $W$ and/or $M$ are manifolds with boundary; on $\partial W, f$ is required to be a (simple) homology equivalence over $Z \pi_{1} W$. A PL embedding of a manifold homotopy equivalent to $M^{n}$, in $W^{n+2}$, determines a Poincaré embedding of $M$ in $W$.

Given a Poincaré embedding $M$ in $W$, as above, with $\pi_{1} M \rightarrow \pi_{1} W$ surjective, one can find a "concordant" Poincaré embedding with $\pi_{1} Y$ a cyclic extension of $\pi_{1} W$ [CS5]. More generally, this can be done if and only if $Z \otimes_{Z\left[\pi_{1} M\right]} Z\left[\pi_{1} W\right] /\left\{[M] \cdot \pi_{2}(W)\right\}$, which is isomorphic to the commutator quotient of the kernel of $\pi_{1} Y \rightarrow \pi_{1} W$, is a cyclic group.

THEOREM 4. Suppose we are given a Poincaré embedding of $M^{n}$ in $W^{n+2}$, 
$n \geqq 3$. If $n$ is odd or if, for $Y$ as above, $\pi_{1} Y$ is isomorphic to $\pi_{1} W$ via the natural map, or if $\pi_{1} W$ vanishes or is infinite cyclic ${ }^{5}$ and $\pi_{1} Y$ is a cyclic extension of $\pi_{1} W$, then this Poincare embedding can be realized by a $P L$ embedding.

Note. If $\pi_{1} M=0$ and $n$ is odd, then for only one class of manifolds $(N, g)$ in the set ht $(M)$, with $g: N \rightarrow M$ a homotopy equivalence, will the composition of the given Poincaré embedding with $g$ be realizable by a locally flat embedding. If $n$ is even and $\pi_{1} M=0$, this composite will be realizable as a PL embedding which is locally flat except at one point for only one class of manifolds $(N, g)$ in the homotopy triangulations $h t(M)$. For $\pi_{1} M \neq 0$, there are examples involving a torus for which no such embedding exists. For $n$ even, the general obstruction to realizing a Poincare embedding by a PL embedding can be described in the terms of the $\Gamma$-groups introduced in [CS2], [CS3], [CS4].

Outline of the proof of Theorem 4. Using induced PL neighborhoods [S1], a classifying space $B R N_{2}\left(B S R N_{2}\right)$ for concordance classes of (oriented) PL codimension two thickenings (regular neighborhoods) of (oriented) manifolds can be constructed. There are natural maps $\mathrm{BSO}_{2} \rightarrow \mathrm{BSRN}_{2} \rightarrow \mathrm{BSG}_{2}=\mathrm{BSO}_{2}$. Let $G_{2} / R_{2}$ be the fiber of $B S R N_{2} \rightarrow$ $\mathrm{BSO}_{2}$. There is a natural map $\mathrm{G}_{2} / R \mathrm{~N}_{2} \rightarrow G / P L$.

An analogous PL embedding theorem for higher codimensions is proved ([H], [W, 11.3]) using the isomorphism $G_{k} / P L_{k}=G / P L, k \geqq 3$, to lift (stable) normal invariants to $G_{k} / P L_{k}$ and an argument showing that a resulting surgery problem can be solved. A related construction using (i) and (iii) of Theorem 5 reduces the argument to showing, using methods of [CS4], that an induced homology surgery problem can be solved. Results of [CS4] applied there to similar problems for locally flat embeddings show that the surgery problem can always be solved when $n$ is odd. For $n$ even, by a further computation $[\mathbf{C S 4}, \mathrm{V}]$ of $\Gamma$-groups, the homology surgery obstruction can be killed by varying on the top cell of $M$ the lift of a normal invariant into $G_{2} / R N_{2}$. Note that a trivial normal invariant can be lifted to a trivial map, for $n$ odd, and to a map trivial in the complement of a point, for $n$ even, for which the homology surgery problem can be solved.

Let $\Gamma^{\varepsilon(k)}, \varepsilon(k)=\operatorname{sign}(-1)^{k}$, denote the groups $\Gamma_{2 k+2}\left(\phi_{0}\right)$ of [CS3], isomorphic to high dimensional knot cobordism groups. Let $\gamma$ be the FoxMilnor cobordism group of knots $S^{1} \subset S^{3}$. Let $Y$ be the fiber of $K(\gamma ; 2) \times K\left(\Gamma_{(2)}^{+} ; 4\right) \rightarrow K\left(Z_{2} ; 4\right)$ given by the cohomology class $\alpha \otimes 1+$ $1 \otimes \beta, \alpha$ the square of the class given by the Arf invariant and $\beta$ given by the mod two reduction of $\frac{1}{8}$ (index). For a group $G$, let $G_{(2)}$ and $G_{(\text {odd) }}$

\footnotetext{
${ }^{5}$ The result remains valid if $\pi_{1} W$ is free abelian and $\pi_{1} Y$ is abelian.
} 
denote localizations at and away from 2 , and $G_{Q}=G \otimes Q$.

THEOREM 6. (i) $\mathrm{BSRN}_{2}$ is homotopy equivalent to $\mathrm{BSO}_{2} \times \mathrm{G}_{2} / \mathrm{RN}_{2}$.

(ii) There is a fiber product diagram

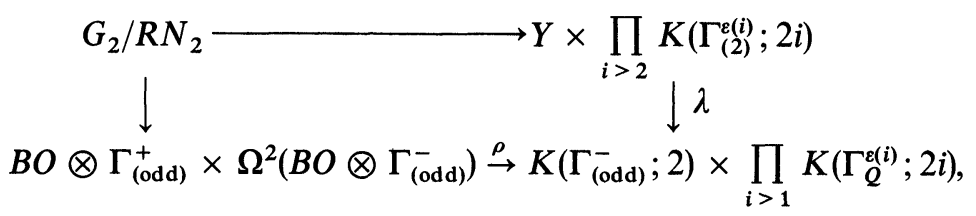

where $\rho$ is essentially the Pontrjagin character and $\lambda$ is induced by natural maps $\gamma \rightarrow \Gamma^{-}$and $\Gamma_{(2)}^{ \pm} \rightarrow \Gamma_{Q}^{ \pm}$.

(iii) The natural map $B S R N_{2} \rightarrow G / P L$ splits (unnaturally).

Results similar to (i) and (ii) were announced by L. Jones [J], "modulo low dimensional complications". The proof is similar to Sullivan's "characteristic variety theorem," which may be interpreted as the description of $G / P L$ associated to the Browder-Novikov surgery theory.

In the present case, surgery theory is replaced by the homology surgery theory of [CS4]. A general "characteristic variety theorem" for suitable periodic spaces $X$, with period 4 and with retractions of bordism groups to homotopy satisfying suitable product formulas, seems to have been known for some time, at least to Morgan and to Jones.

The Eilenberg obstruction to contracting a map to $G_{2} / R N_{2}$ are identified with the Noguchi obstructions $[\mathbf{N}]$ to making an embedding locally flat.

Due to the absence of a simple description of the $\mathrm{H}$-structure of $B S R N_{2}$, (i) requires careful geometry.

\section{REFERENCES}

[CS1] S. E. Cappell and J. L. Shaneson, Topological knots and knot cobordism, Topology (to appear).

[CS2] - Submanifolds, group actions, and knots. I, Bull. Amer. Math. Soc. 78(1972), $1045-1048$.

[CS3] - Submanifolds, group actions, and knots. II, Bull. Amer. Math. Soc. 78(1972), $1049-1052$.

[CS4] - The codimension two placement problem and homology equivalent manifolds (to appear).

[CS5] , (to appear).

[CS6] Codimension two submanifolds, Proc. 1972 Strasburg conference on Topology and Analysis, Ann. Inst. Fourier (to appear).

$[\mathbf{H}]$ A. Haefliger, Knotted spheres and related geometric problems, Proc. Internat. Congress Math. (Moscow, 1966), "Mir", Moscow, 1968, pp. 437-445. MR 39 \#6325.

$[\mathbf{J}]$ L. Jones, Three characteristic classes measuring the obstruction to P.L. local unknottedness, Bull. Amer. Math. Soc. 78 (1972), 979-980.

[KM] M. Kato and Y. Matsumoto, Simply-connected surgery of submanifolds in codimension two (to appear).

[N] H. Noguchi, Obstructions to locally flat embeddings of combinatorial manifolds, 
Topology 5 (1966), 203-213. MR 33 \# 724.

[S1] D. Stone, Polyhedral topology, Lecture Notes in Math., vol. 252, Springer-Verlag, Berlin and New York, 1972.

[S2] D. Sullivan, Geometric topology, Seminar Notes, Princeton University, Princeton, N.J., 1967 (mimeographed notes).

[W] C. T. C. Wall, Surgery of compact manifolds, Academic Press, New York, 1970.

Department of Mathematics, Princeton University, Princeton, New Jersey 08540

Weizman Institute of SCIENCE, Rehovot, ISRAEL

Department of Mathematics, Rutgers University, New Brunswick, New Jersey 08903 